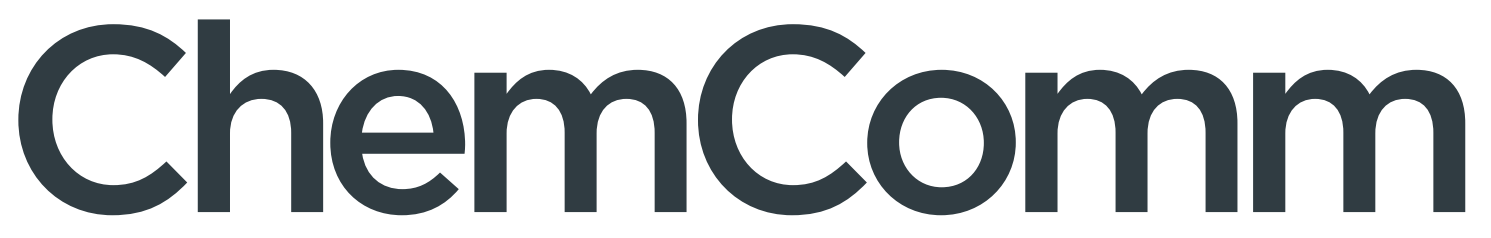

Chemical Communications

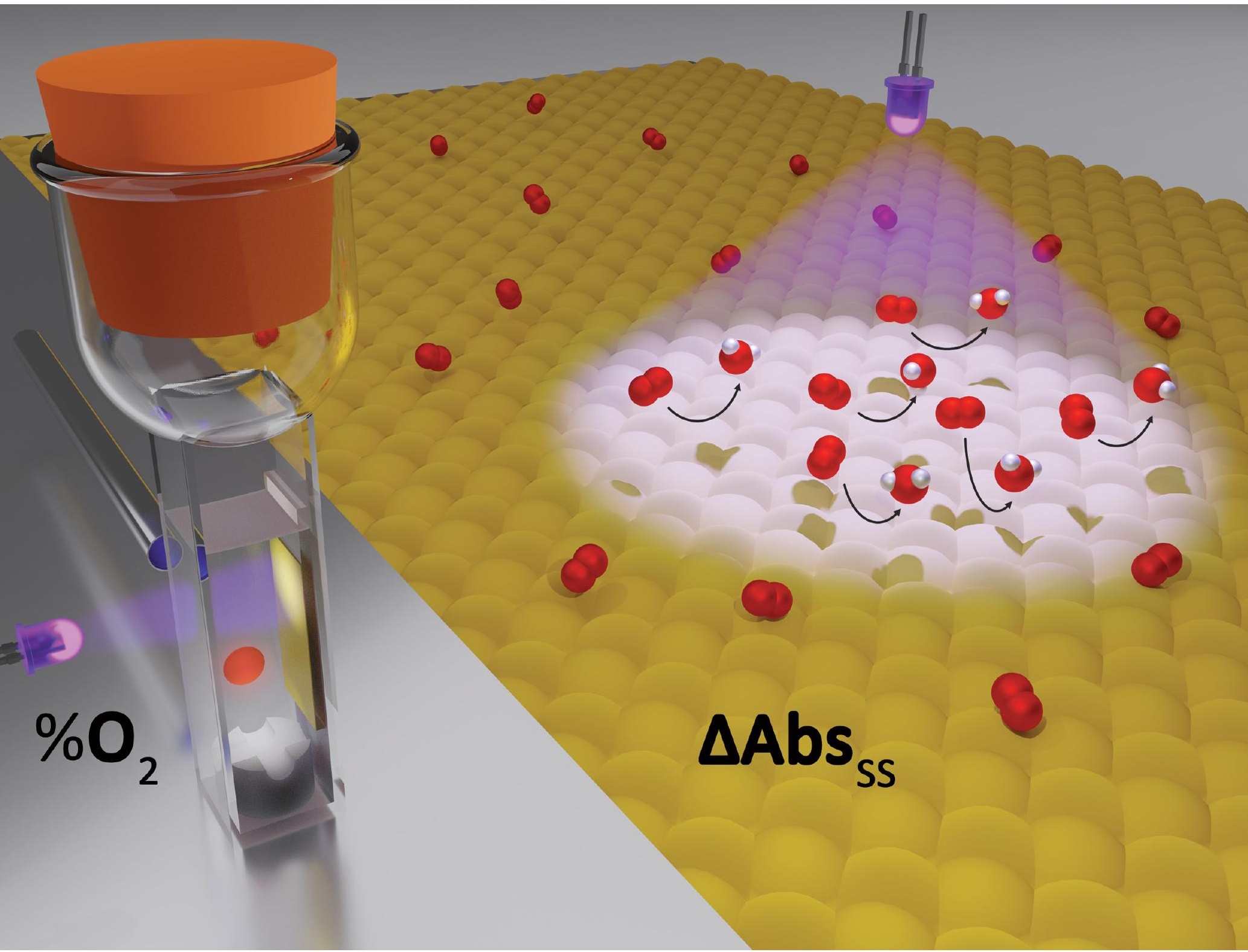


D) Check for updates

Cite this: Chem. Commun., 2021, 57, 1591

Received 18th December 2020, Accepted 26th January 2021

DOI: $10.1039 / \mathrm{d} 0 \mathrm{cc} 08208 \mathrm{~b}$

rsc.li/chemcomm

\section{Kinetics of the photocatalysed reduction of oxygen by CdS probed using photoinduced absorption spectroscopy (PIAS) $\dagger$}

\author{
Aaron McNeill, Christopher O'Rourke and Andrew Mills (D) *
}

\begin{abstract}
Photoinduced absorption spectroscopy, PIAS, is used to determine the order of reaction and so identify the rate determining step in the reduction of $\mathrm{O}_{2}$ by a sacrificial electron donor, photocatalysed by a nanoparticular film of CdS. This is the first report on the use of PIAS to probe the kinetics of photocatalysis exhibited by a non-oxide semiconductor.
\end{abstract}

Cadmium sulfide (CdS) is one of the most commonly employed, visible-light absorbing semiconductor photocatalysts $\left(E_{\mathrm{bg}}=2.4 \mathrm{eV}^{1}\right)$ due to the highly reducing nature of its photogenerated conductance band electrons $\left(E_{\mathrm{CB}}\left(\mathrm{e}^{-}\right)=-0.93 \mathrm{~V}\right.$ vs. NHE at $\mathrm{pH} 7^{2}$ ) and the highly oxidising nature of its photogenerated valence band holes $\left(E_{\mathrm{CB}}\left(\mathrm{h}^{+}\right)=1.47 \mathrm{~V} v\right.$ s. NHE at $\left.\mathrm{pH} 7^{2}\right)$. Unfortunately, CdS, like most visible-light absorbing photocatalysts, undergoes photoanodic corrosion, i.e. ${ }^{3}$

$$
2 \mathrm{~h}^{+}+\mathrm{CdS} \rightarrow \mathrm{Cd}^{2+}+\mathrm{S} \downarrow
$$

particularly in air-saturated, aqueous solution, since dissolved oxygen is an effective scavenger of photogenerated electrons, i.e.:

$$
\mathrm{e}^{-}+\mathrm{O}_{2} \rightarrow \mathrm{O}_{2}^{-}
$$

where superoxide, $\mathrm{O}_{2}{ }^{-}$, is subsequently further reduced to water, ${ }^{4-6}$ leaving the photogenerated holes to drive reaction (1). However, despite this feature, CdS is often used to photocatalyse reduction reactions with electron acceptors such as, water, ${ }^{7,8}$ organics, ${ }^{9,10}$ oxygen $^{11}$ and metal ions, ${ }^{7,8,12}$ but without exhibiting signs of photocorrosion; this is achieved by adding a sacrificial electron donor (SED), such as EDTA, ${ }^{13}$ cysteine ${ }^{14}$ ascorbic acid/ascorbate, ${ }^{15}$ or sodium sulfide, ${ }^{16}$ which reacts efficiently and irreversibly with the photogenerated holes, thereby preventing reaction (1), via the following reaction,

$$
\mathrm{h}^{+}+\mathrm{SED} \rightarrow \mathrm{SED}^{+}
$$

Queen's University Belfast, School of Chemistry and Chemical Engineering, David Keir Building, Stranmillis Road, Belfast, BT9 5AG, UK.

E-mail: andrew.mills@qub.ac.uk

$\dagger$ Electronic supplementary information (ESI) available. See DOI: 10.1039/ d0cc08208b where $\mathrm{SED}^{+}$is the oxidised form of the SED that decomposes irreversibly. When the electron acceptor in question is oxygen, the overall photocatalytic process can be summarised as follows:

$$
\mathrm{O}_{2}+\mathrm{SED} \underset{h \geq 2.4 \mathrm{eV}}{\stackrel{\mathrm{CdS}}{\longrightarrow}} \mathrm{O}_{2}^{-}+\mathrm{SED}^{+}
$$

Recently, photoinduced absorption spectroscopy, PIAS, coupled with transient photocurrent (TC) measurements i.e., PIAS/TC, has been used to probe the kinetics of water oxidation exhibited by different semiconductor oxide photoanodes, such as $\mathrm{Fe}_{2} \mathrm{O}_{3}, \mathrm{TiO}_{2}$ and $\mathrm{BiVO}_{4} \cdot{ }^{17-19}$ In these PIAS/TC studies the optical absorption, $\Delta \mathrm{Abs}_{\mathrm{ss}}$, exhibited by photogenerated holes accumulated at the surface of the semiconductor photoanode and the photocurrent, $J_{\mathrm{ss}}$, are monitored simultaneously under ultra-bandgap, steady-state irradiation conditions and a large anodic bias. The latter ensures that the steady-state level of photogenerated electrons is so low that the reaction of the surface-accumulated photogenerated holes can be considered to be due to the oxidation of water exclusively. In all the PIAS/ TC studies reported to date the values of $\Delta \mathrm{Abs}_{\mathrm{ss}}$ and $J_{\mathrm{ss}}$, are measured as a function of incident irradiance and the order of reaction of the photogenerated holes taken as the value of the gradient of the plot $\log \left(J_{\mathrm{ss}}\right)$ vs. $\log \left(\Delta \mathrm{Abs}_{\mathrm{ss}}\right)$.

PIAS has never been used to probe a photocatalysed reduction reaction by an n-type semiconductor, nor probe a non-oxide photocatalyst. However, interestingly, it has been shown that nanoparticulate CdS exhibits a blue shift in its absorption edge upon electronic excitation. The effect increases with incident irradiance and decreasing particle size. This shift in absorption edge is usually interpreted as a Burstein shift i.e., an increase in the optical band gap due to the population of the conduction band with photogenerated electrons. ${ }^{20-24}$ Liu and Bard also found the same shift can be produced by an excess of holes. $^{25}$

In studies of this effect ${ }^{21,23}$ using CdS colloids, the kinetics of decay of the resulting flash-induced change in absorption are complex and short-lived, i.e. $<12 \mathrm{ns,} \mathrm{but} \mathrm{this} \mathrm{lifetime} \mathrm{is}$ 
increased markedly when a SED is present. Thus, Albery et al. ${ }^{24}$ in a microsecond flash photolysis study of a CdS colloid (17 $\mathrm{nm}$ particles) in the presence of a SED (0.02 $\mathrm{M} \mathrm{Na} \mathrm{Na}_{2} \mathrm{~S}$ ), reported a half-life of $c a .50 \mathrm{~ms}$ in the transient bleaching due to a blue shift in its absorption spectrum! These workers also found that, in the additional presence of methyl viologen, which acts as an electron acceptor, there exists a direct correlation between the kinetics of decay in the transient absorbance, $\Delta \mathrm{Abs}$, and those for the concomitant photogeneration of the reduced methyl radical, ${ }^{24}$ which suggests that $\Delta \mathrm{Abs}$ is directly related to the concentration of the surface accumulated (i.e. trapped) conductance band electrons, $\left[\mathrm{e}^{-}\right]$, photogenerated by the flash. This feature is exploited in this PIAS-based study of reaction (4), photocatalysed by CdS.

The CdS films on microscope glass used in this work were made from a CdS nanopowder (Sigma Aldrich; average particle size $c a .50 \mathrm{~nm}$ ) using a modified version of a method reported by Ito et al. for making screen-printed $\mathrm{TiO}_{2}$ films ${ }^{26}$ details of which are given in S1 in the ESI, $\dagger$ file. The thickness of the dried film was $c a$. $1.1 \mu \mathrm{m}$, yellow and slightly opalescent in appearance, as illustrated by the photograph of the CdS film in Fig. 1(a). The absorption spectrum of the film, illustrated in Fig. 1(b), revealed an absorbance onset at ca. $534 \mathrm{~nm}$, i.e. $2.3 \mathrm{eV}$, which corresponds closely with the known bandgap of $2.4 \mathrm{eV}$ for CdS. ${ }^{1}$

In almost all previously reported PIAS studies, the semiconductor photocatalysis films were photoanodes and the steadystate photocurrent taken as a measure of the rate of the photocatalysed oxidation reaction. This approach allows the order of the photoelectrochemical oxidation to be determined, provided the faradaic efficiency is unity, which is not always the case. ${ }^{27}$ In contrast, in this work a quite different approach was employed in the application of PIAS; one in which, under steady-state irradiation conditions, the measured value of $\Delta \mathrm{Abs}_{\mathrm{ss}}$ due to the surface-accumulated photogenerated electrons, $\left[\mathrm{e}^{-}\right]_{\mathrm{ss}}$, was related to the actual, independently measured rate of the photocatalytic process, and not a photocurrent. In addition, the values of $\Delta \mathrm{Abs}_{\mathrm{ss}}$ and rates were not varied by altering the incident irradiance, $\rho$, as is the usual practice, but rather by changing the reactant concentration. In this work the photocatalytic reaction probed in this way was the reduction of oxygen by an SED, photocatalysed by CdS, i.e. Reaction (4), where $\mathrm{SED}=0.1 \mathrm{M}$ solution of $1: 1$ molar ratio sodium ascorbate and ascorbic acid (NaA/AA). The latter was selected
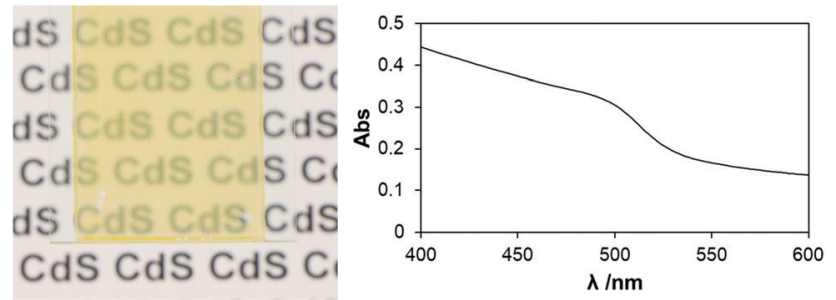

Fig. 1 (a) Photograph and (b) UV/Vis absorption spectrum of the CdS nanoparticulate film.

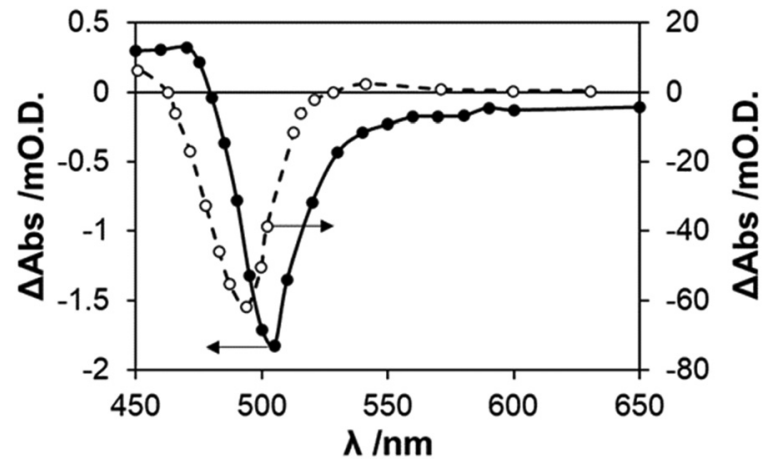

Fig. 2 Transient bleaching in the difference absorption spectrum exhibited by CdS as (a) a colloid, no SED, 10 ms after flash ${ }^{24}$ (broken line) and (b) the CdS nanoparticle film on glass, measured using PIAS, with a NaA/AA SED $(0.1 \mathrm{M})$ and $\rho=29.3 \mathrm{~mW} \mathrm{~cm}{ }^{-2}, 365 \mathrm{~nm}$, solid line. Both solutions were $\mathrm{O}_{2}$ free.

as the SED, since a previous study reported it to be highly effective in preventing the photocorrosion of CdS in an air-saturated solution. ${ }^{15}$ Details of the PIAS system used in this work are given in $\mathrm{S} 2$ in the ESI, $\uparrow$ which was used to determine the variation in the steady-state irradiation induced change in absorbance, $\Delta \mathrm{Abs}_{\mathrm{ss}}$ exhibited by a CdS nanoparticulate film in $20 \mathrm{~mL}$ of an Ar-purged, 0.1 M NaA/AA aqueous solution, with

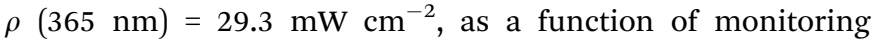
wavelength, $\lambda_{\mathrm{m}}$. The results of this work are illustrated in Fig. 2 and reveal a very similar spectral profile to that reported by Albery et al. for a CdS colloid, ${ }^{24}$ although with an absorption maximum at 505, rather than $485 \mathrm{~nm}$, and with a smaller shift in absorption spectrum ( $c a .0 .8$ instead of $3 \mathrm{~nm}$ ). The spectral differences are probably due to the differences in CdS particle size, i.e. $17 c f .50 \mathrm{~nm}$.

In a subsequent PIAS study of the CdS film carried out using $\lambda_{\mathrm{m}}=505 \mathrm{~nm}$ in aqueous solution with $0.1 \mathrm{M} \mathrm{NaA} / \mathrm{AA}$, and $\rho(420 \mathrm{~nm})=15.0 \mathrm{~mW} \mathrm{~cm}{ }^{-2}$, the change in absorbance, i.e. $\Delta \mathrm{Abs}$, was recorded before during and after steady state irradiation in the presence of different saturation levels of dissolved $\mathrm{O}_{2}$ spanning the range 0 to $21 \%$; the results of this work are illustrated in Fig. 3(a). The plot of the data generated from this work in the form of $\Delta \mathrm{Abs}_{\mathrm{ss}} v s$. $\% \mathrm{O}_{2}$, illustrated in Fig. 3(b), shows that the steady-state transient absorbance, $\Delta \mathrm{Abs}_{\mathrm{ss}}$, due to photogenerated conductance band electrons, decreases in magnitude with increasing $\% \mathrm{O}_{2}$. As illustrated by the solid line in Fig. 3(b), the variation in $\Delta \mathrm{Abs}_{\mathrm{ss}} v s$. $\% \mathrm{O}_{2}$ fits an equation with the following form,

$$
\Delta \mathrm{Abs}_{\mathrm{ss}}(\text { in mO.D. })=\frac{\alpha}{\beta \% \mathrm{O}_{2}+1}
$$

where $\alpha$ and $\beta$ are fitting constants with best fit values of 2.9 and $0.035 \% \mathrm{O}_{2}{ }^{-1}$, respectively. An explanation for this variation is provided by a kinetic analysis of the measured dark decays which are shown in greater detail in Fig. S3(a) in the ESI. $\dagger$ The value of $\Delta \mathrm{Abs}$ (dark) at any time during the decay process is assumed to be a measure of the dark concentration of the surfaceaccumulated photogenerated conductance band electrons, $\left[\mathrm{e}^{-}\right]$. 

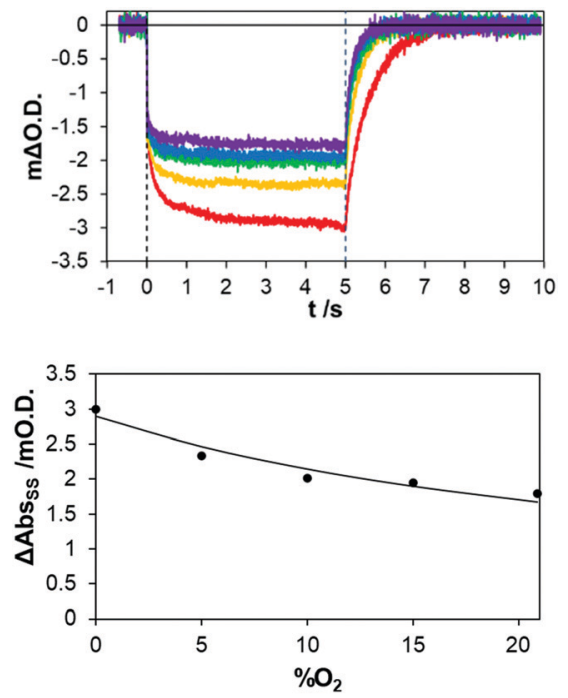

Fig. 3 (a) Plot of $\Delta$ Abs vs. time for a $C d S$ film, recorded before during and after steady state irradiation $\rho=15.0 \mathrm{~mW} \mathrm{~cm}{ }^{-2}, 420 \mathrm{~nm}$, with NaA/AA $(0.1 \mathrm{M})$, and in the presence of dissolved $\mathrm{O}_{2}$ maintained at different saturation levels ranging from 0 to $21 \%$; the $\% \mathrm{O}_{2}$ saturation levels used were (from bottom to top): 0, 5, 10, 15 and 21\%, respectively; (b) plot of $\Delta \mathrm{Abs}_{\mathrm{ss}}$ vs. $\% \mathrm{O}_{2}$ constructed using data from (a), with a solid line of best fit based on eqn (5), with $\alpha$ and $\beta$ equal to 2.9 and $0.035 \% \mathrm{O}_{2}{ }^{-1}$, respectively.

All of the $\Delta \mathrm{Abs}$ (dark) $v s$. time decays exhibited first order kinetics, from which different first order rate constant, $k_{1}$, values could be extracted. A plot of $k_{1} v$ s. $\% \mathrm{O}_{2}$ illustrated in Fig. S3(b) in the ESI, $\dagger$ reveals a good straight line, which fits the following expression,

$$
k_{1}=k_{\mathrm{O} 2} \% \mathrm{O}_{2}+k_{0}
$$

with $k_{\mathrm{O} 2}$ and $k_{0}$ best fit values of $0.175\left(\% \mathrm{O}_{2}\right)^{-1} \mathrm{~s}^{-1}$ and $1.88 \mathrm{~s}^{-1}$, respectively. The latter value equates to $k_{\mathrm{O} 2}=1.35 \times 10^{4} \mathrm{M}^{-1} \mathrm{~s}^{-1}$ for $k_{\mathrm{O} 2}$ which compares favourably with the value of $2.0 \times 10^{4} \mathrm{M}^{-1} \mathrm{~s}^{-1}$, reported by others in their study of $\mathrm{O}_{2}$ reduction by $\mathrm{TiO}_{2}{ }^{28}$

The value of $k_{0}$, the first order rate constant for the decay of the photogenerated electrons in the absence of $\mathrm{O}_{2}$, is large, as indicated by the associated dark decay curve (red) illustrated in Fig. 3(a). This large value for $k_{0}$, suggests that NaA/AA is not very effective hole scavenger in this system, so that either many of the photogenerated holes are trapped, e.g. as lattice bond $\mathrm{S}^{-}$ radicals, or more likely, its oxidised form, dehydroascorbic acid, or intermediate, is able to efficiently back-react with surface accumulated photogenerated electrons. These results indicate that, despite the presence of an SED, electron-hole recombination is a dominant dark reaction, making $k_{0}$ large, and reducing the reduction of $\mathrm{O}_{2}$ to the role of a relatively minor side reaction. Evidence that this is indeed the case for the CdS films comes from a simple study of the variation of $\Delta \mathrm{Abs}_{\mathrm{ss}}$ as a function of irradiance, the results for which are illustrated in Fig. 4 and reveal a direct dependence of $\Delta \mathrm{Abs}_{\mathrm{ss}}$ upon $\rho^{0.5}$. The same dependence of $\Delta$ Abs upon $\rho^{0.5}$ was found by Albery et al. in their flash photolysis study of a CdS colloid in the presence of $0.05 \mathrm{M}$ cysteine as the SED and is taken there

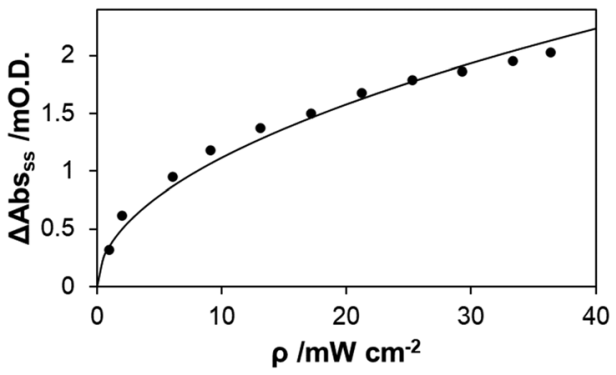

Fig. 4 Plot of the measured variation in $\Delta \mathrm{Abs}_{\mathrm{ss}}$ for a $\mathrm{CdS}$ film as a function of incident irradiance, $\rho$, with all other conditions as in Fig. 2. The solid line of best fit has been calculated assuming $\Delta \mathrm{Abs}_{\mathrm{ss}}$ is $\propto \rho^{0.5}$.

and here as indicative that electron-hole recombination dominates the fate of the photogenerated electron-hole pairs. ${ }^{24}$

As illustrated by Fig. S3(b) in the ESI, $\dagger$ and eqn (6), the above study also shows that there is a direct dependence of $k_{1}$ upon $\% \mathrm{O}_{2}$, which indicates that, although reaction (4) is a minor process, the rate-determining step, rds, in the reduction of $\mathrm{O}_{2}$ by photogenerated electrons on the CdS film particles is first order with respect to $\Delta \mathrm{Abs}$ (i.e. $\left[\mathrm{e}^{-}\right]$), which strongly suggests that it is reaction (2), the initial production of superoxide that is the rds. This finding and conclusion are consistent with those made by others studying the reduction of $\mathrm{O}_{2}$ by photogenerated conductance band electrons on $\mathrm{TiO}_{2}{ }^{28}$

In the CdS film/SED system, given the above, it follows that under steady-state irradiation conditions the rate of photogeneration of surface accumulated conductance band electrons, which is proportional to $\rho^{0.5}$, will be matched by the rate of their loss, so that,

$$
\delta \rho^{0.5}=\left(k_{\mathrm{O}_{2}} \% \mathrm{O}_{2}+k_{0}\right) \Delta \mathrm{Abs}_{\mathrm{ss}}\left(\operatorname{or}\left[\mathrm{e}^{-}\right]_{\mathrm{ss}}\right)
$$

where $\delta$ is a proportionality constant. Reassuringly, the above steady-state equation is consistent with the $\Delta \mathrm{Abs}_{\mathrm{ss}} v s . \% \mathrm{O}_{2}$ plot illustrated in Fig. 3(b) and described by eqn (5) since the two equations, eqn (5) and (7), are mathematically equivalent assuming $\alpha=\delta \rho^{0.5} / k_{0}$ and $\beta=k_{\mathrm{O}_{2}} / k_{0}$.

If reaction (2) is the rate determining step in the photocatalysed reduction of $\mathrm{O}_{2}$, then the rate of reduction of $\mathrm{O}_{2}, r$, will be equal to $k_{\mathrm{O}_{2}} \% \mathrm{O}_{2}\left[\mathrm{e}^{-}\right]_{\mathrm{ss}}$. If the latter term is combined with eqn (7) the following expression can be derived,

$$
r=\frac{\gamma\left(\% \mathrm{O}_{2}\right) \rho^{0.5}}{\beta\left(\% \mathrm{O}_{2}\right)+1}
$$

where $\gamma=k_{\mathrm{O}_{2}} \delta / k_{0}$ and $\beta=k_{\mathrm{O}_{2}} / k_{0}$. Interestingly, this form of the rate equation is very similar to the empirical, apparent Langmuir-Hinshelwood rate equation, which is so often used to fit the kinetics of photocatalysis exhibited by most powder photocatalysts in the photocatalysed mineralisation of a test pollutant, $\mathrm{P}$, by dissolved $\mathrm{O}_{2}$, i.e.

$$
\mathrm{d}[\mathrm{P}] / \mathrm{d} t=k^{*}\left(\frac{K_{\mathrm{P}}[\mathrm{P}]}{1+K_{\mathrm{P}}[\mathrm{P}]}\right)\left(\frac{K_{\mathrm{O}_{2}}\left[\mathrm{O}_{2}\right]}{1+K_{\mathrm{O}_{2}}\left[\mathrm{O}_{2}\right]}\right)
$$

where $k^{*}$ is the maximum rate for a fixed irradiance, $\rho$, and $K_{\mathrm{P}}$ and $K_{\mathrm{O}_{2}}$ are apparent Langmuir adsorption coefficients. Usually, in studies of the photocatalytic mineralisation of 


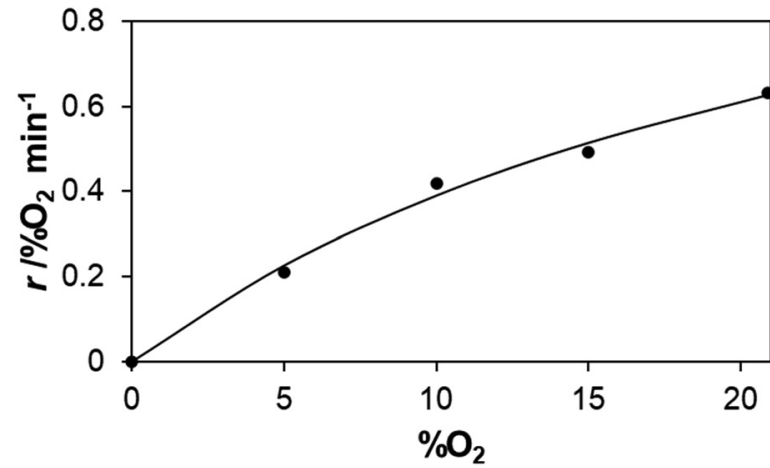

Fig. 5 Measured initial rate of the reduction of $\mathrm{O}_{2}$ by $0.1 \mathrm{M} \mathrm{NaA} / \mathrm{AA}$, photocatalysed by a CdS film, irradiated with $420 \mathrm{~nm} \mathrm{LED}\left(30 \mathrm{~mW} \mathrm{~cm}{ }^{-2}\right.$ ), as a function of the $\% \mathrm{O}_{2}$ used to initially saturate the solution. The solid line fit to the data is based on eqn (8) with $\gamma . \% \mathrm{O}_{2} \rho^{0.5}=1.42 \% \mathrm{O}_{2} \min ^{-1}$ and $\beta=0.038 \% \mathrm{O}_{2}{ }^{-1}$.

organics the concentration of $\mathrm{O}_{2}$ is fixed and high, by sparging the reaction solution continuously with $\mathrm{O}_{2}$, and the rate of change in $[\mathrm{P}]$ is monitored as a function of the initial concentration of $[\mathrm{P}] \cdot{ }^{29}$ Less common are studies of the same system, such as here, where the level of pollutant (i.e. the SED in this work) is fixed and high and the rate of $\mathrm{O}_{2}$ reduction is determined as a function of $\% \mathrm{O}_{2} \cdot{ }^{29,30}$ Encouragingly, under such conditions, eqn (9) reduces to the same form as eqn (8), with the difference that the fitting constants in eqn (9) are replaced with parameters, such as $\rho, k_{\mathrm{O}_{2}}$ and $k_{0}$, that are related to a particular reaction mechanism and rate determining step.

In order to test the validity of eqn (8), derived from the PIAS study of the CdS film in $0.1 \mathrm{M} \mathrm{NaA} / \mathrm{AA}$, the variation of the rate of reduction of $\mathrm{O}_{2}, r$, was measured as a function of $\% \mathrm{O}_{2}$, using a set up that was very similar to that used in the PIAS study, with the exception that the system was sealed and the dissolved level of $\mathrm{O}_{2}$ was measured using an $\mathrm{O}_{2}$ xyDot $^{\circledR}{ }^{\circledR}$ OxySense $^{31}$ ). Further details of the steady-state irradiation system are given in S4 in the ESI. $\dagger$ The results of this work are illustrated in Fig. 5 with a solid line of best fit based on eqn (8) with $\gamma . \% \mathrm{O}_{2} \rho^{0.5}=1.42 \% \mathrm{O}_{2} \mathrm{~min}^{-1}$ and $\beta=0.038 \% \mathrm{O}_{2}{ }^{-1}$. Reassuringly, the latter value $\left(\beta=0.038 \% \mathrm{O}_{2}{ }^{-1}\right)$ is near identical to the value of $\beta=0.035 \% \mathrm{O}_{2}{ }^{-1}$ reported earlier, see Fig. 3(b), and eqn (5), despite the fact that they were derived by two very different methods, namely from $\Delta \mathrm{Abs}_{\mathrm{ss}}$ (PIAS) and initial rate $(r)$ measurements. The strong similarity in the two values for $\beta$ provides further support that the rate of reduction of $\mathrm{O}_{2}$ in reaction (4) by the $\mathrm{CdS}$ films used in this work, wit $h$ the $\mathrm{SED}=0.1 \mathrm{M} \mathrm{NaA} / \mathrm{AA}$, is first order with respect to the steady-state, concentration of surface-accumulated photogenerated electrons, $\left[\mathrm{e}^{-}\right]_{\mathrm{ss}}$, making it likely that reaction (2) is the rate determining step.

In conclusion, PIAS, combined with steady-state reaction rate studies, can be used to probe the reduction of $\mathrm{O}_{2}$ by a SED, photocatalysed by a film comprised of CdS nanoparticles. This is the first report of the use of PIAS to study the kinetics of photocatalysis of a non-oxide semiconductor and the first use of PIAS to study a photocatalysed reduction reaction. This work suggests that PIAS can be used more widely to provide invaluable mechanistic information for many different photocatalyst materials, including non-oxides, in powder form.

\section{Conflicts of interest}

There are no conflicts to declare.

\section{Notes and references}

1 F. Boakye and D. Nusenu, Solid State Commun., 1997, 102, 323-326.

2 A. Mills, N. Wells and C. O'Rourke, J. Photochem. Photobiol., A, 2017, 338, 123-133.

3 D. Meissner, R. Memming, B. Kastening and D. Bahnemann, Chem. Phys. Lett., 1986, 127, 419-423.

4 G. Mattioli, F. Filippone and A. A. Bonapasta, J. Am. Chem. Soc., 2006, 128, 13772-13780.

5 F. Filippone, G. Mattioli and A. A. Bonapasta, Catal. Today, 2007, 129, 169-176.

6 E. T. Wahyuni and N. H. Aprilita, Photoreduction Processes over TiO2 Photocatalyst, 2018, 129-145.

7 M. Berr, A. Vaneski, A. S. Susha, J. Rodríguez-Fernández, M. Döblinger, F. Jäckel, A. L. Rogach and J. Feldmann, Appl. Phys. Lett., 2010, 97, 093108.

8 D. W. Wakerley, M. F. Kuehnel, K. L. Orchard, K. H. Ly, T. E. Rosser and E. Reisner, Nat. Energy, 2017, 2, 17021.

9 T. Uekert, M. F. Kuehnel, D. W. Wakerley and E. Reisner, Energy Environ. Sci., 2018, 11, 2853-2857.

10 Y. Shiraishi, M. Katayama, M. Hashimoto and T. Hirai, Chem. Commun., 2018, 54, 452-455.

11 A. E. Raevskaya, A. L. Stroyuk and S. Y. Kuchmii, J. Nanopart. Res., 2004, 6, 149-158.

12 Y. Zhang, Y. Zhao, Z. Xu, H. Su, X. Bian, S. Zhang, X. Dong, L. Zeng, T. Zeng, M. Feng, L. Li and V. K. Sharma, Appl. Catal., B, 2020, 262, 118306.

13 A. Mills and A. Green, J. Photochem. Photobiol., A, 1991, 59, 199-208.

14 J. R. Darwent and G. Porter, J. Chem. Soc., Chem. Commun., 1981, 4, 145-146.

15 A. McNeill and A. Mills, J. Phys. Energy, 2020, 2, 044003.

16 A. J. Nozik, Appl. Phys. Lett., 1977, 30, 567-569.

17 F. Le Formal, E. Pastor, S. D. Tilley, C. A. Mesa, S. R. Pendlebury, M. Grätzel and J. R. Durrant, J. Am. Chem. Soc., 2015, 137, 6629-6637.

18 Y. Ma, C. A. Mesa, E. Pastor, A. Kafizas, L. Francàs, F. Le Formal, S. R. Pendlebury and J. R. Durrant, ACS Energy Lett., 2016, 1, 618-623.

19 A. Kafizas, Y. Ma, E. Pastor, S. R. Pendlebury, C. Mesa, L. Francàs, F. Le Formal, N. Noor, M. Ling, C. Sotelo-Vazquez, C. J. Carmalt, I. P. Parkin and J. R. Durrant, ACS Catal., 2017, 7, 4896-4903.

20 E. Burstein, Phys. Rev., 1954, 93, 632-633.

21 P. V. Kamat, N. M. Dimitrijevic and A. J. Nozik, J. Phys. Chem., 1989, 93, 2873-2875.

22 C.-Y. Liu and A. J. Bard, J. Phys. Chem., 1989, 93, 3232-3237.

23 P. V. Kamat, T. W. Ebbesen, N. M. Dimitrijevic and A. J. Nozik, Chem. Phys. Lett., 1989, 157, 384-389.

24 W. J. Albery, G. T. Brown, J. R. Darwent and E. Saievar-Iranizad, J. Chem. Soc., Faraday Trans. 1, 1985, 81, 1999-2007.

25 C.-Y. Liu and A. J. Bard, J. Phys. Chem., 1989, 93, 7749-7750.

26 S. Ito, P. Chen, P. Comte, M. K. Nazeeruddin, P. Liska, P. Péchy and M. Grätzel, Prog. Photovoltaics, 2007, 15, 603-612.

27 C. A. Mesa, L. Francàs, K. R. Yang, P. Garrido-Barros, E. Pastor, Y. Ma, A. Kafizas, T. E. Rosser, M. T. Mayer, E. Reisner, M. Grätzel, V. S. Batista and J. R. Durrant, Nat. Chem., 2020, 12, 82-89.

28 H. H. Mohamed, R. Dillert and D. W. Bahnemann, J. Photochem. Photobiol., A, 2011, 217, 271-274.

29 A. Mills, C. O'Rourke and K. Moore, J. Photochem. Photobiol., A, 2015, 310, 66-105.

30 A. Mills and J. Wang, Z. Phys. Chem., 1999, 213, 49-58.

31 OxySense, https://oxysense.com, accessed December 2020. 